\title{
Graph based discrete optimization in structural dynamics
}

\author{
B. BLACHOWSKI ${ }^{1}$ and W. GUTKOWSKI ${ }^{2 *}$ \\ ${ }^{1}$ Institute of Fundamental Technological Research of the Polish Academy of Sciences, 5b Pawinskiego St., 02-106 Warsaw, Poland \\ ${ }^{2}$ Institute of Mechanized Construction and Rock Mining, 6/8 Racjonalizacji St., 02-673 Warsaw, Poland
}

\begin{abstract}
In this study, a relatively simple method of discrete structural optimization with dynamic loads is presented. It is based on a tree graph, representing discrete values of the structural weight. In practical design, the number of such values may be very large. This is because they are equal to the combination numbers, arising from numbers of structural members and prefabricated elements. The starting point of the method is the weight obtained from continuous optimization, which is assumed to be the lower bound of all possible discrete weights. Applying the graph, it is possible to find a set of weights close to the continuous solution. The smallest of these values, fulfilling constraints, is assumed to be the discrete minimum weight solution. Constraints can be imposed on stresses, displacements and accelerations. The short outline of the method is presented in Sec. 2. The idea of discrete structural optimization by means of graphs. The knowledge needed to apply the method is limited to the FEM and graph representation.

The paper is illustrated with two examples. The first one deals with a transmission tower subjected to stochastic wind loading. The second one with a composite floor subjected to deterministic dynamic forces, coming from the synchronized crowd activities, like dance or aerobic.
\end{abstract}

Key words: discrete structural optimization, combinatorial optimization, structural dynamics, stochastic loading, problem oriented optimization, graphs.

\section{Introduction}

So far, most of research on the minimum weight structural design has been carried out on structures subjected to static loads. Only in the sixties and seventies, of the past century, first works on structural optimization with constraints imposed on eigenvalues were published. Turner [1] discussed a numerical procedure for finding minimum weight of a complex structure under a constraint imposed on a given natural frequency. Tang [2] presented an optimum design of threemember frame subjected to frequency constraints. However, only few of them, among others by Mroz [3] dealt with constraint resulting from dynamic loadings. Most of attention has been paid to problems with small number of degrees of freedom, and with steady-state sinusoidal responses. An extended survey by Pierson [4] covering over sixty papers, gives profound information about progress, at this time, done in the field of structural optimization with dynamic constraints.

With the increasing computational possibilities and knowledge of mathematical programming, it was possible to extend engineering design to more practical problems. McGee and Phan [5] considered space frames with relatively larger numbers of degrees of freedom and dynamic constraints. Their discussion, based on the Optimality Criteria approach, was devoted to the computational complexity of problems with constraints imposed on several natural frequencies. The paper is illustrated with eight-member frame, and structural weight concentrated on four rigid joints with six degrees of freedom each.

The increased demands for research in structural and machine dynamics optimization were caused, mostly by devel- opment of aircraft industry. In the review paper Grandhi [6], widely cited to this day, presents an extended discussion on variety problems related to structural optimization with frequency constraints. He turns attention, to one of the most important problem, consisting in the switching of vibration modes due to structural size modification.

In the last decade, several stochastic approaches has been used for dynamic structural optimization problems. Most of them are based on the Genetic Algorithm, a heuristic approach giving near optimum solutions. A profound review on discrete stochastic methods in structural optimization is given by Arora et al. [7]. However, with the lack of the mathematical background of the algorithm, the distance of the solution from real extreme is unknown. An example of minimum weight design with constraints imposed on several natural frequencies, obtained by genetic algorithm, is presented in the paper by Lee et al. [8]. An extended review of discrete structural optimization is given by Gutkowski [9]. One of the simplest heuristic methods for finding minimum structural weight, by removing redundant material, is presented by Blachowski and Gutkowski [10].

The increasing requirements for structural safety caused farther demands for solutions of structural response to dynamic loads. However, due the complexity of dynamic analysis, in some papers we don't find a direct dynamic analysis, but analysis under a static load equivalent, to a dynamic one. Kang et al. [11] proposed structural optimization approach under equivalent static load, generating identical displacements as caused by dynamic excitations. Choi et al. [12] presented a method, based on modal analysis, transforming dynamic loads into equivalent static one.

\footnotetext{
*e-mail: wgutkows@ippt.pan.pl
} 
In general, civil engineering structures are subjected, to a large extent, to stochastic loads. These are mainly loads coming from wind or earthquake, and their stochastic nature cannot be neglected in the design. Recently, then, more attention has been paid to this type of problems. Papadrakakis et al. [13] discussed multi-objective optimization problem of skeletal structures under seismic condition, applying Evolutionary Algorithm.

Norkus and Karkauskas [14] presented an algorithm for truss optimization under stochastic load, composed of three parts. Each of them consists of separate problems. In the first one, variation bounds of stresses and displacements for a given time period are presented. Next, an analysis for defined bounds is performed. The third one is optimizing the member cross-section areas, satisfying the safety constraints. All three problems are applied in each optimization cycle. Jensen and Beer [15] proposed discrete-continuous structural optimization under stochastic load, applying generalized Optimality Criteria method, including dual function for discrete variables. An adaptive genetic algorithm for structural topology optimization of transmission tower, with discrete sizing, was proposed by Guo and Li [16] The problem is solved for static loads only.

A concept of applying graphs to discrete structural optimization was proposed by Gutkowski et al. [17]. It is based on an algorithm, elaborated by Iwanow [18], for finding an ordered sequence of values of a discrete linear function. The basic assumption made in the method is that the continuous minimum of structural weight constitutes a lower bound for discrete solution. However, the mentioned algorithm gives only structural volumes, without information on member forces. It means that the obtained, from the graph, discrete solution should be checked if physical constraints are not violated. The algorithm allows for arbitrary numbers of permutations of graph branches, representing member volumes. The idea of the method is illustrated, by a simple problem, in Sec. 2. Now, the algorithm presented by Blachowski and Gutkowski [19] is extended to structure composed of trusses, frames and plates, under dynamic conditions.

The algorithm is divided in two parts. In the first part, using the graph property, described in Subsec. 3.2, the values of the discrete cost function, larger than continuous one, but next to it are found. The algorithm starts with a tree graph containing only two branches. In the second part, the obtained combination of structural members is verified for constraints.

The same procedure is performed, after enlarging the catalogue to four, six etc. available parameters.

In this study, the application of the discussed graph approach is extended to structures under dynamic loads. Two practical problems are discussed. The first one is a transmission tower under stochastic wind load. A transmission tower can be considered, under static load, as a pin connected. However, the pin joint assumption can't be applied in structural dynamics. Blachowski and Gutkowski [20] presented several structures for which significant differences between eigenfrequencies and even eigenmodes could be observed. It depends if the structure is considered as a truss (pin joints), or as a frame (rigid joints). Considering dynamics of the transmission tower as a frame requires division of each structural member in several FE. This increases significantly number of degrees of freedom, entering in the problems. To overcome difficulties with very large numbers of DOF, a load-dependent Ritz vector method, proposed by $\mathrm{Gu}$ et al. [21] is applied. Due to the wind nature, the method allows to reduce the number of DOF to be considered from thousand to less than twenty only.

The second problem deals with a composite floor, which consists of concrete slab, supported by a set of steel beams. The floor is subjected to dynamic forces coming from the synchronized crowd activities, like dance or aerobic. In this case the load is considered to be deterministic.

In both examples, in the structural analysis, an algorithm of FEM, based on an earlier work by Blachowski [22] is applied.

In the paper, as mentioned above, tree graphs are used for finding the minimum from a very large number of combinations. The latter arises from the number of structural members and available parameters located in a catalogue of prefabricated elements. In order to be more consistent in applying graphs, one could expect the application of the graph based physical systems modelling approach. However, the experience in the application of the method is, so far, limited to the problems with very small numbers of elements. Borutzky [23] is giving examples with two degrees of freedom, and a one loop electrical circuit. A. Buchacz [24] is discussing free vibrations of a single beam. These examples show lack of usefulness of the method in cases of systems with hundreds elements, like the structures considered in the paper.

\section{The idea of discrete structural optimization by means of graphs}

In this study, the discrete minimum weight design consists in assigning to each structural member a profile from a given list (catalogue). Here, structures are composed of beams and/or plates and sheets. It must be pointed out, that "assigning" means choosing a set of values of cross section area (CSA), pipes diameters, principal moment of inertia (PMI) etc. from a catalogue containing dozens or even hundreds of profiles. On the other hand, the optimized structure can be also composed of dozens or hundreds of elements.

Denoting by $k=$ the number of all available, in a catalogue, elements, and by $j=$ the number of all structural members, or linking groups, design consists in searching for an optimum from among $n=k^{j}$ combinations. Even, in a relatively simple engineering problem, there can be 10 structural members and 10 available parameters, which give $10^{10}$ combinations. More cases, when several catalogues are taken into consideration, together with different structural profiles (beams and plates), are discussed in Sec. 3.

Searches for discrete minimum weight consists in verifications of fulfilment of state equations and imposed constraints for each combination. In many practical designs, as mentioned above, $n$ is so large, that a simple enumeration searching for 
optimum is impossible, even using very powerful numerical tools.

This is the reason, that only applying a heuristic approach it is possible find optimum, or more rigorously, near optimum.

An outline of the proposed method is presented on a graph in Fig. 1, showing it for a structure composed of a beam and a plate. The beam can be chosen from three available parameters, and the plate from two different thicknesses. Subscripts are indicating position of the parameters in the catalogue, say CSA. Superscripts indicate structural member numbers.

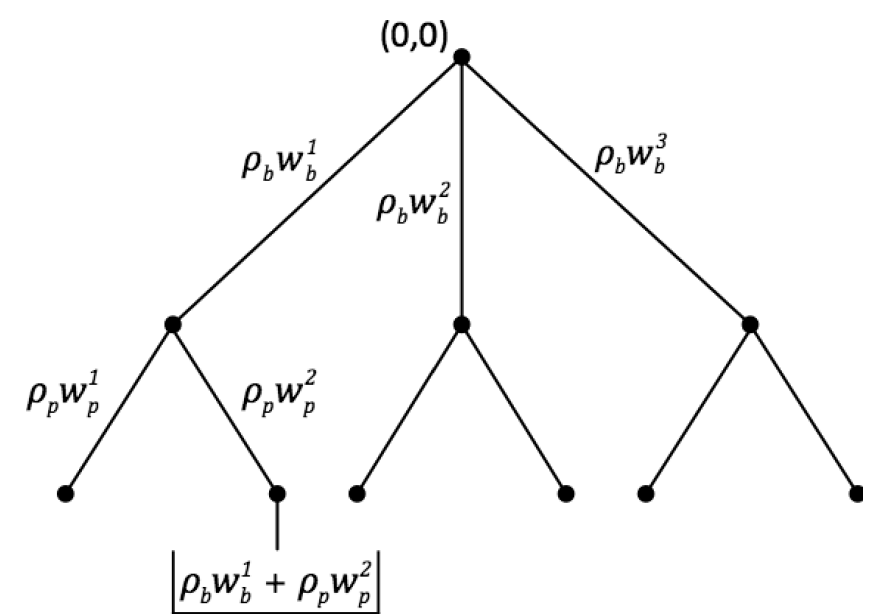

Fig. 1. Graph with three available parameters for a beam and two for a plate

The edges of the graph are representing the structural weights of beams and plates respectively. They are chosen from catalogues of parameters, which are ordered with their increasing values. Vertices are representing sum of weights of structural members represented by edges starting from $(0,0)$ vertex. This way, at the bottom of the graph, vertices are representing all possible combination between structural members and available parameters.

Inspecting the given graph, it can be seen, that extreme vertex, belonging to the same layer of the graph (subgraph), represent the smallest and the largest volumes from all possible volumes included in the layer. This important property of the discussed graph (subgraph) will be used in finding the discrete minimum of structural volume.

A large number of discussed combinations can be omitted from considerations, by applying an assumption that minimum of the discrete structural weight $\bar{W}_{d}$ cannot be smaller than minimum of the continuous structural weight $\bar{W}_{c}$ This can be noted as $\bar{W}_{c} \leq \bar{W}_{d}$. This assumption comes from the observation of a discretized continuous function.

If $\bar{W}_{c}$ is smaller than the sum of all smallest structural weights, it means, that structural elements with smallest available parameters are candidates for discrete minimum weight. The minimum can be confirmed only after verification, if imposed on the structure constraints are not violated.

On the other hand, if $\bar{W}_{c}$ is larger than the value at the vertex being most to the right side of the graph, the solution within assumed available parameters doesn't exist. Finally, if
$\bar{W}_{c}$ is between two extreme vertices, a part of the graph can be neglected from farther considerations. This is when values at the vertices are smaller than continuous minimum weight.

The values of structural weight represented by vertices of the last layer of the graph are not ordered according to their increasing values. Their order depends on the order of subsequent graph branches. This is the reason for which, in the cases with large numbers of combinations, a set of graphs with permuted branches are considered.

Detailed and formal presentation of this problem is presented in Sec. 3.

\section{The algorithm for discrete minimum weight structural design}

Let us present in details, the algorithm outlined in the previous section. As motioned there, the discrete optimum problem consists in finding the minimum weight from all values arising from all combinations relating structural members and available parameters. In practical design, structural members are divided in groups having the same sectional parameters. In general, the number of combinations occurring in a structure composed of $b^{0}$ different beams and $p^{0}$ different plates is equal to the following product:

$$
\left(\prod_{b=1}^{b^{0}} k_{b}^{0}\right)\left(\prod_{p=1}^{p^{0}} j_{p}^{0}\right)
$$

where $k_{b}^{0}$ and $j_{p}^{0}$ are numbers of, available in catalogues, beam sections and plate thicknesses respectively.

Before proceeding with farther considerations, let introduce the following, principal notations: $b=\left[1,2, \ldots, b^{0}\right]$ and $p=\left[1,2, \ldots, p^{0}\right]$ is the number of a beam and/or of a plate in the structure respectively, $n_{b}=\left[n_{1}, n_{2}, n_{3}, \ldots, n_{b^{0}}\right]$ the number of beam structural members in $b$-th linking group, $m_{b}=\left[m_{1}, m_{2}, m_{3}, \ldots, m_{p^{0}}\right]$ the number of plate structural members in $p$-th linking group, $L_{b}-$ sum of $n_{b}$ beam lengths $l_{b, n}$ in $b$-th linking group, $L_{b}=\sum_{n=1}^{n_{b}} l_{b, n}, S_{p}-$ sum of $m_{p}$ plate areas $s_{p, m}$ in $p$-th linking group, $S_{p}=\sum_{m=1}^{m_{p}} s_{p, m}, A_{b}$, $D_{b}, t_{b}, I_{b}$ - cross section area, diameter, thickness and moment of inertia of a pipe respectively, of $b$-th beam, $A_{b}, H_{b}, h_{b}$ - cross section area, height and flange thickness of a $I$ - beam respectively, of $b$-th beam, $h_{p}$ - the thickness of $p$-th plate, $v_{b}$ - volume of $b$-th beam linking group $v_{b}=A_{b} L_{b}, v_{p}$ - volume of $p$-th plate linking group, $v_{p}=h_{p} S_{p}, k_{b}=\left[1,2,3, \ldots, k_{b}^{0}\right]$, $j_{p}=\left[1,2,3, \ldots, j_{p}^{0}\right]$ the number of a beam profile and plate thickness from the catalogue, assigned to $b$-th beam or $b$-th linking group, or to $p$-th plate or $p$-th linking group, respectively, $\bar{A}_{b} \ldots$ over line defines section parameters obtained from continuous solution, $A_{b}^{k_{b}} \ldots$ superscript $k_{b}$ defines the parameter from the catalogue assigned to the $b$-th structural element, $\bar{A}_{b}^{k_{b}} \ldots$ over line with a superscript $k_{b}$-th define the parameter from the catalogue, assigned to the $b$-th structural element, obtained from discrete minimum solution, $V, V_{b}, V_{p}$ - total structural volume, volume of all beam elements, vol- 
ume of all plate elements respectively, $\bar{V}_{c}, \bar{V}_{d}-$ continuous and discrete minimum volumes, respectively, $\bar{W}_{c}, \bar{W}_{d}-$ continuous and discrete minimum weights, respectively, $\rho_{b}, \rho_{p}-$ density of beam and plate materials, respectively.

3.1. Graph representing structural weight. The discussed graph is a directed tree graph. An edge of the graph is labelled with the weight of the structural member. A vertex is labelled with a pair of numbers and with the sum of all edges joining the vertex with the graph root $(0,0)$. At the bottom of the graph, vertices $\left(j_{0}, k_{j}\right)$ represent all possible structural weights, arising from combinations between structural members and available parameters.

The applied directed tree graph and all its transformation belong to well established graph theory [25].

As mentioned above, the algorithm is based on an assumption that the minimum structural weight $\bar{W}_{c}$, obtained from continuous optimization, constitutes the lower bound of all possible discrete values of structural weight $W_{d}$

$$
\bar{W}_{c} \leq W_{d}=\rho_{b} \sum_{b=1}^{b^{0}} v_{b}^{k_{b}}+\rho_{p} \sum_{p=1}^{p^{0}} v_{p}^{k_{p}} .
$$

The algorithm tends to approach the discrete value, as close as possible, to the continuous one, under the condition $\bar{V}_{c} \leq V_{d}$. The first try is along path OCD (Fig. 2). The next is along OEF. Both of them are giving discrete values larger than continuous one. The third try along OGH shows that $\mathrm{H}$ vertex value is smaller than continuous minimum. It means that all other branches at left from GH can be neglected from farther considerations. Now, the aim is still to approach closer to $\bar{V}_{c}$. This can be done, going along EIJK. In this case, the discrete structural weight in the closest to $\bar{V}_{c}$, and at the same time larger than it. If the structure composed of such a set of available cross sections area is fulfilling given constraints, it is considered as discrete minimum volume $\bar{V}_{d}$.

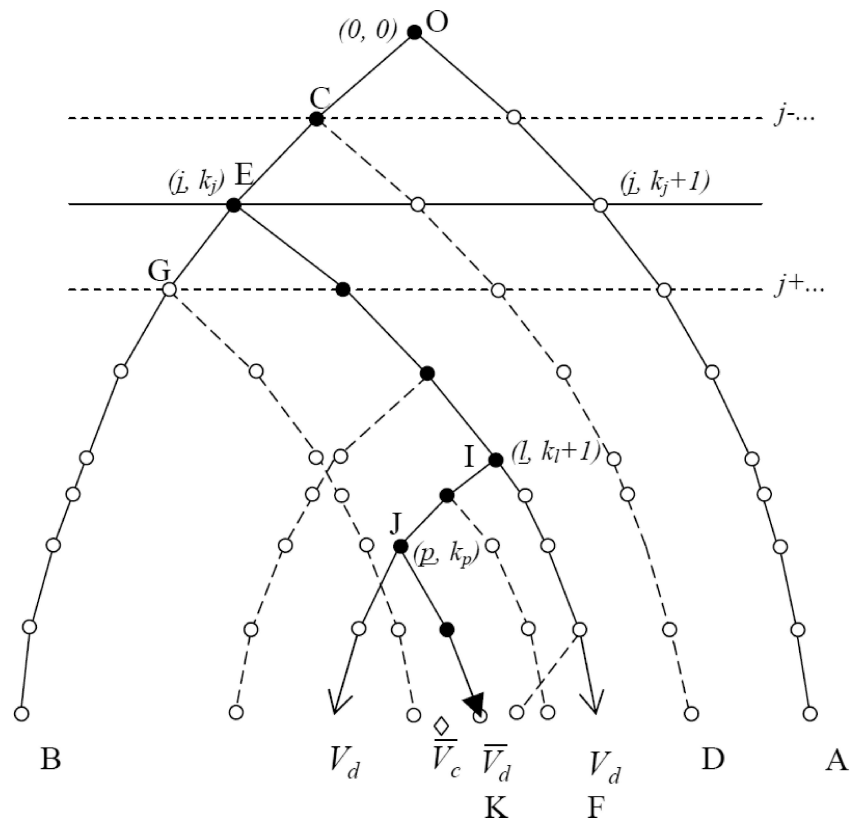

Fig. 2. Search for smallest feasible discrete cost function
3.2. The graph algorithm. The algorithm is composed of two parts. In the first one, discrete cost function values, closest to the continuous minimum weight are found. In the second one, verification of constraints in discrete solutions, obtained in the first part, is performed.

PART 1: Find value of the discrete cost function $W_{d} \geq \bar{W}_{c}$

\section{STEP 1.1}

Find continuous minimum weight $\bar{W}_{c}$ of the given structure, subjected to the assumed loading conditions and constraints.

\section{STEP 1.2}

Take, for each structural member, or linking group, two subsequent values:

for a beam

$$
A_{b}^{k_{b}} \leq \bar{A}_{b} \leq A_{b}^{k_{b}+1}
$$

with

$$
D_{b}^{k_{b}} \leq \bar{D}_{b} \leq D_{b}^{k_{b}+1} ; \quad t_{b}^{k_{b}} \leq \bar{t}_{b} \leq t_{b}^{k_{b}+1}
$$

and/or

$$
\begin{gathered}
H_{b}^{k_{b}} \leq \bar{H}_{b} \leq H_{b}^{k_{b}+1} \\
h_{b}^{k_{b}} \leq \bar{h}_{b} \leq h_{b}^{k_{b}+1} ; \quad I_{b}^{k_{b}} \leq \bar{I}_{b} \leq I_{b}^{k_{b}+1}
\end{gathered}
$$

and for a plate

$$
h_{p}^{k_{p}} \leq \bar{h}_{p} \leq h_{p}^{k_{p}+1} .
$$

Set a list of parameters, composed of two, just mentioned, values for all structural members.

\section{STEP 1.3}

Construct two branch graph (Fig. 2) and:

(i) Find the smallest values of the structural weight, $W_{j, \max }^{k_{j}}$, represented by extreme vertices of subgraphs with roots $\left(j, k_{j}\right)$, for $j=1,2, \ldots,\left(j^{0}-1\right)$. Save the subgraph with $\operatorname{root}\left(\underline{j}, k_{j}\right)$, giving the smallest value of $\left(W_{j, \max }^{k_{j}}-\bar{W}_{c}\right)$ for $W_{j, \max }^{k_{j}}>\bar{W}_{c}$.

(ii) Find the largest values of the structural weight $W_{l, \min }^{k_{j+1}}$, represented by extreme vertices of subgraph with roots $\left(l, k_{l}+1\right)$, for $l=j,(j+1) \ldots\left(j^{0}-1\right)$. Save the subgraph with the $\operatorname{root}^{-}\left(\underline{l}, k_{l}+1\right)$, giving the smallest of $\left(W_{l, \text { min }}^{k_{j+1}}-\bar{W}_{c}\right)$ for $W_{l, \text { min }}^{k_{j+1}}>\bar{W}_{c}$.

(iii) Find the smallest values of the structural weight, $W_{p, \max }^{k_{p}}$, represented by extreme vertices of subgraphs with roots $\left(p, k_{p}\right)$, for $p=\underline{l},(\underline{l}+1) \ldots\left(j^{0}-1\right)$. Save the subgraph with root $\left(p, k_{p}\right)$, giving the smallest value of $\left(W_{p, \max }^{k_{p}}-\bar{W}_{c}\right)$ for $W_{p, \max }^{k_{p}}>\bar{W}_{c}$.

(iv) Go to (ii) and (iii), increasing subsequently $l$ and $p$ to $\left(j^{0}-1\right)$.

(v) Save, from all $m$ arrangements, structures with values of discrete cost function, giving $\min \left(W_{d}-\bar{W}_{c}\right)$.

\section{STEP 1.4}

Assume an arbitrary number $m$. Select randomly $m$ different permutations of graph edges, represented by the index $j$. 
Larger $m$ is, the probability of obtaining better result increases. Preform STEP 1.3 for each permutation.

\section{PART 2: Verify constraint violation}

Find, for all $m$ structures obtained in STEP 1.3 (v), a parameter

$$
\mu(1)=\max \left(q_{\max , i} / u^{0}, \sigma_{j} / \sigma^{0}, \quad \ddot{q}_{\max , i} / a^{0}\right)
$$

for $i=1,2, \ldots, i^{0}$ and $j=1,2, \ldots, j^{0}$.

If $\mu \leq 1$, the structure is considered as of minimum weight $\bar{W}_{d}$. If several discrete solutions are fulfilling this condition, the one with the smallest weight is considered as the discrete minimum weight.

If all of $m$ structures are not fulfilling this condition, go to PART 1, STEP 1.2 of the algorithm. Enlarge lists of available profiles to four positions and proceed with all steps there.

The above algorithm can be also presented in the form of block diagram (Fig. 3), as follows:

$j=1,2, \ldots, j^{0}$ (tree level)

$k=1,2, \ldots, k^{0}$ (tree branch, for two branch tree $k^{0}=2$ )

$\bar{V}_{c}$ (continuous minimum volume)

$\bar{V}_{d}$ (discrete minimum volume) vel)

$V_{d, j}$ (discrete volume obtained, starting from $j$-th tree le-

$$
V_{d, j}^{k}=\sum_{r=1}^{j-1} A_{r}^{k_{r}} l_{r}+A_{j}^{k} l_{j}+\sum_{r=j+1}^{j^{0}} A_{r}^{k^{0}} l_{r} .
$$

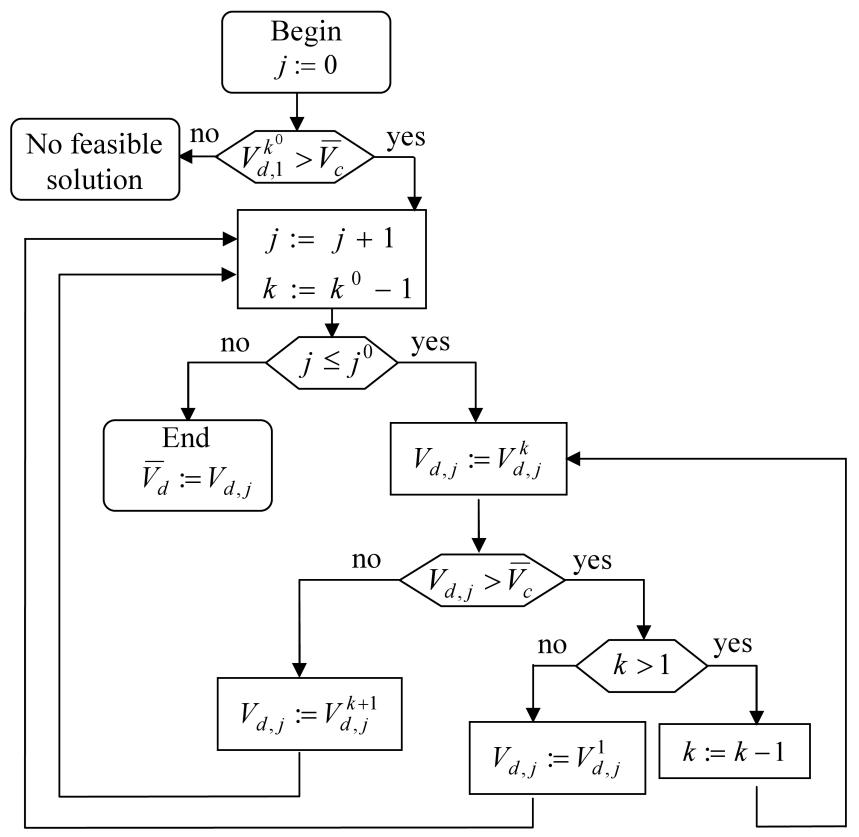

Fig. 3. Block diagram of the algorithm

\section{Discrete minimum weigh design of a transmission tower under wind load}

The first example illustrating the application and validity of the proposed method is devoted to a 27 meter high transmission tower (Figs. 4 and 5). The tower is supporting conductors and ground-wires. The basic data for the structure are taken from the Guidelines for Electrical Transmission Line Structural Loading the $3^{\text {rd }}$ ed., published by ASCE. Spans between neighbouring towers are equal to 200 meters. The static shape of the wires and conductors is assumed to be a catenary curve with a six-meter sag. Initial tension in wires equals $7 \mathrm{kN}$. a)

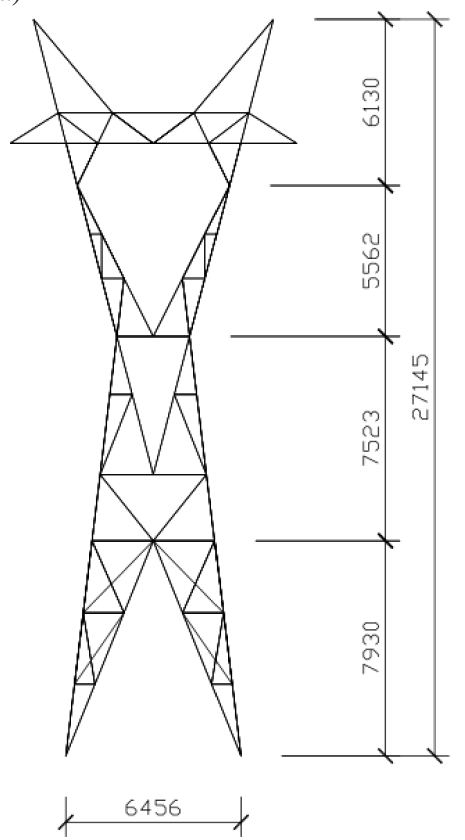

b)

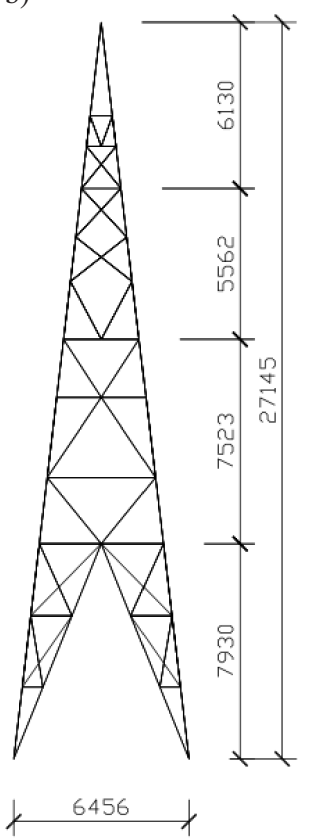

Fig. 4. Transmission tower data - plane XZ (a), plane YZ (b) a)

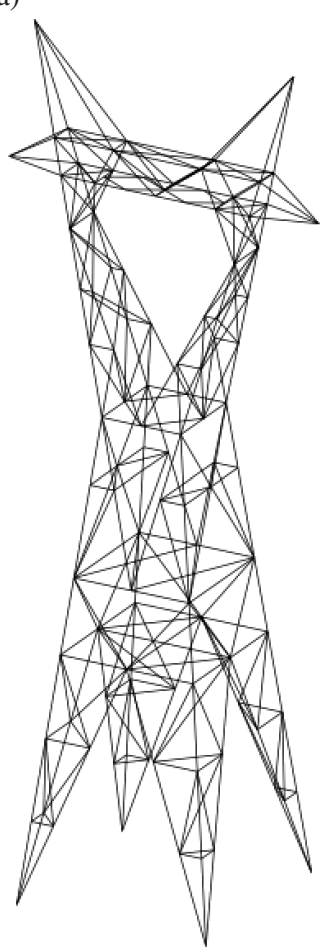

b)

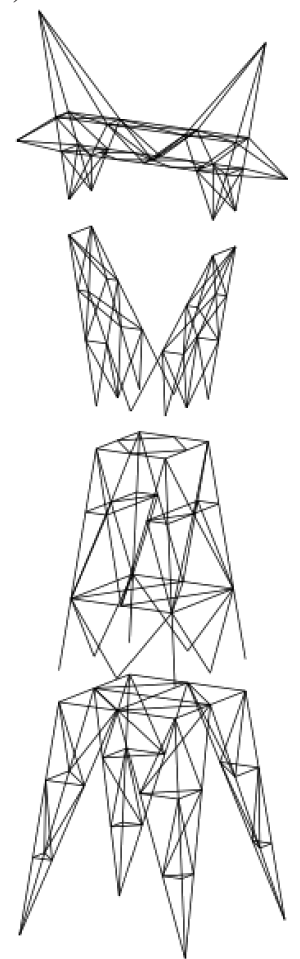

Fig. 5. 3D view of the tower (a), tower divided into four assembling modules (b) 
It is assumed that structural members are made of pipes, rigidly connected at joints. The $3 \mathrm{D}$ structure is divided in four sections (Fig. 5), and members are linked in seven groups of the same cross section areas. A commercially available catalogue of circular hollow sections, consisting of 314 sections from CHS 22/1.2 to CHS 273/8 is considered. Constraints are imposed on both, stresses and displacements.

The structure is subjected to forces, arising from stochastic wind, blowing horizontally in a direction perpendicular to wires.

4.1. Structural response to the dynamic load. In the following dynamic response analysis, the classical FEM motion equations, with $N D O F$ degrees of freedom are applied:

$$
\mathbf{M} \ddot{\mathbf{q}}_{t o t a l}(t)+\mathbf{C} \dot{\mathbf{q}}_{\text {total }}(t)+\mathbf{K} \mathbf{q}_{\text {total }}(t)=\mathbf{p}_{\text {total }}(t)
$$

where $\mathbf{M}, \mathbf{C}, \mathbf{K}$ are mass, damping and stiffness matrices respectively $(N D O F \times N D O F)$. The number $N D O F$ includes the numbers of structural joints, together with joints, arising from partition of each structural member in four FE. $\ddot{\mathbf{q}}_{t o t a l}$, $\dot{\mathbf{q}}_{\text {total }}, \mathbf{q}_{\text {total }}$ are vectors of total structural node accelerations, velocities and displacements, respectively $(N D O F \times 1)$. $\mathbf{p}_{\text {total }}$ is an external, stochastic force vector $(N D O F \times 1)$. It is also assumed that the total displacement can be considered as a sum of constant static displacements $\overline{\mathbf{q}}$, equal to the mean value of total displacements, and fluctuating parts $\mathbf{q}(t)$

$$
\mathbf{q}_{\text {total }}(t)=\overline{\mathbf{q}}+\mathbf{q}(t) .
$$

The fluctuating part of the dynamic equation, has, under this assumption the following form

$$
\mathbf{M} \ddot{\mathbf{q}}(t)+\mathbf{C} \dot{\mathbf{q}}(t)+\mathbf{K q}(t)=\mathbf{p}(t) .
$$

In the problem, relatively a large number of modes are orthogonal to the horizontal wind direction, and their contribution to the structural dynamic response can be neglected. Taking into account the above statement, one can extract from the system of Eqs. (7), a set of NMode «NDOF, effective subset of modes shapes, called Ritz vectors, proposed by Wilson [26].

Applying Ritz vectors the structural displacement vector $\mathbf{q}(t)$ can be transformed into

$$
\mathbf{q}(t)=\mathbf{\Phi}_{R} \eta(t)
$$

where $\boldsymbol{\Phi}_{R}$ is the modal transformation matrix $(N D O F \times$ NMode) defined by a designer, and $\eta(t)$ modal vector. Here, $\boldsymbol{\Phi}_{R}$ is assumed to be a matrix $(2106 \times 24)$.

Substituting (8) in (7) we get

$$
\mathbf{M}_{R} \ddot{\eta}(t)+\mathbf{C}_{R} \dot{\eta}(t)+\mathbf{K}_{R} \eta(t)=\boldsymbol{\Phi}_{R}^{T} \mathbf{p}(t)
$$

where

$$
\mathbf{M}_{R}=\boldsymbol{\Phi}_{R}^{T} \mathbf{M} \boldsymbol{\Phi}_{R}, \quad \mathbf{C}_{R}=\boldsymbol{\Phi}_{R}^{T} \mathbf{C} \boldsymbol{\Phi}_{R}, \quad \mathbf{K}_{R}=\boldsymbol{\Phi}_{R}^{T} \mathbf{K} \boldsymbol{\Phi}_{R} .
$$

In the end the dynamics of the transmission tower is represented by 24 mode shapes (Figs. 6 and 7).
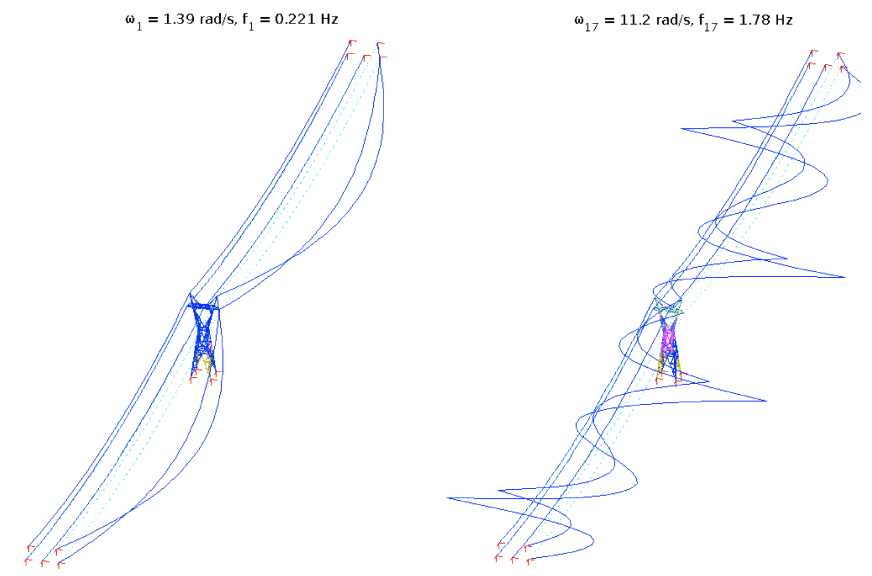

Fig. 6. Two mode shapes of the transmission system - first mode (a), seventeenth (b)
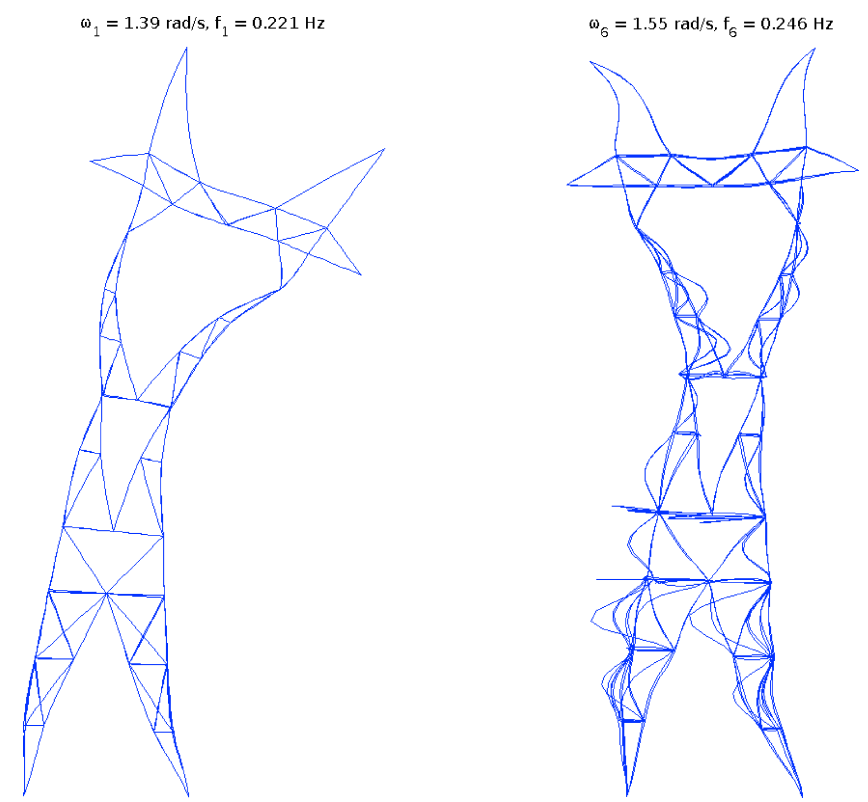

Fig. 7. Tower mode shapes - first mode (a), sixth mode (b)

4.2. Stochastic wind load and structural displacements. The formulae, related to stochastic wind forces and displacements, applied in the problem, are based on the monograph by Buchholdt [27].

The horizontal wind velocity is varying with height above the ground, starting with the velocity equal to $30 \mathrm{~m} / \mathrm{sec}$ at 10 meters above the ground level. The wind power spectrum density is Kaimal type function.

The total wind force $\mathbf{p}_{\text {total }}(t)$ acting on the structure can be expressed in the form

$$
\begin{gathered}
\mathbf{p}_{\text {total }}(t)=\frac{1}{2} \rho_{a i r} \mathbf{C}_{d} \circ \mathbf{A}_{\text {proj }} \circ \\
\circ\left(\mathbf{v}_{\text {total }}(t)-\dot{\mathbf{q}}(t)\right) \circ\left(\mathbf{v}_{\text {total }}(t)-\dot{\mathbf{q}}(t)\right),
\end{gathered}
$$

where $\rho_{\text {air }}$ - air density, $\mathbf{C}_{d}$ - vector of drag coefficients, $\mathbf{A}_{\text {proj }}$ - vector of structure's areas exposed to wind projected in normal direction, $\mathbf{v}_{\text {total }}(t)$ - total wind velocity, o denotes Hadamard product (entrywise product). 
Assuming that the total wind velocity $\mathbf{v}_{\text {total }}(t)$ is a sum of a constant part $\overline{\mathbf{v}}$ and fluctuating one $\mathbf{v}(t)$, and neglecting small nonlinear terms, one gets

$$
\begin{gathered}
\mathbf{p}_{\text {total }}(t)=\frac{1}{2} \rho_{a i r} \mathbf{C}_{d} \circ \mathbf{A}_{\text {proj }} \circ \\
\circ(\overline{\mathbf{v}} \circ \overline{\mathbf{v}}+2 \overline{\mathbf{v}} \circ \mathbf{v}(t)-2 \overline{\mathbf{v}} \circ \dot{\mathbf{q}}(t)) .
\end{gathered}
$$

The first term of Eq. (12) describes static loading

$$
\overline{\mathbf{p}}=\frac{1}{2} \rho_{a i r} \mathbf{C}_{d} \circ \mathbf{A}_{\text {proj }} \circ \overline{\mathbf{v}} \circ \overline{\mathbf{v}} .
$$

The second one describes dynamic loading due to wind fluctuations

$$
\mathbf{p}(t)=\rho_{\text {air }} \mathbf{C}_{d} \circ \mathbf{A}_{\text {proj }} \circ \overline{\mathbf{v}} \circ \mathbf{v}(t) .
$$

For a number of adjoining FEM joints, the wind velocity may have the same value. Denoting it by $w_{\text {total, } j \text { for }}$ $j=[1,2, \ldots, N$ Wind $]$, with $N$ Wind $\ll N D O F$, the relation with $v_{\text {total,i }}$ for $i=[1,2, \ldots, N D O F]$ can be define as $v_{\text {total }, i}=b_{i j} w_{\text {total }, j}$ or in a matrix form

$$
\mathbf{v}_{\text {total }}(t)=\mathbf{B w}_{\text {total }}(t) \text {. }
$$

Together, it was assumed that the spatial wind turbulence acts at 7 wind sections ( 3 along the height of tower, and 1 on each of 4 windward wires). It means, that all total velocities $w_{\text {total }, j}$ for $j=[1,2, \ldots, N W i n d]$ constitute a vector with NWind $=7$ components.

Finally, dynamic wind loading (14) can be expressed in matrix notation as

$$
\mathbf{p}(t)=\mathbf{G} \mathbf{w}(t),
$$

where $\mathbf{G}$ is the load pattern matrix.

The third term of (12) is the aerodynamic damping, usually, for practical calculations a simplified its form is applied, as follows

$$
\rho_{a i r} \mathbf{C}_{d} \circ \mathbf{A}_{\text {proj }} \circ \overline{\mathbf{v}} \circ \dot{\mathbf{q}}(t) .
$$

After reducing the number of equations of motion - applying equations (8)-(10) - the response of the structure due to fluctuating wind velocity can be efficiently calculated using frequency domain method. Based on the paper Carassale et al. [28] the power spectral density matrix of modal coordinates can be evaluated from

$$
\mathbf{S}_{\eta}(f)=\mathbf{H}(f) \boldsymbol{\Phi}_{R}^{T} \mathbf{G S}_{w}(f) \mathbf{G}^{T} \boldsymbol{\Phi}_{R} \mathbf{H}(f)^{*},
$$

where $f$ is the frequency, $\mathbf{H}(f)$ is the frequency response matrix, and $\mathbf{H}(f)^{*}$ is its complex conjugate.

Assuming weak correlation between individual modes, one can simplify the above matrix equation to mode-by-mode form. Following Strommen [29], this can be done by calculating only diagonal terms of the spectral density matrix. Knowing spectral of individual modal coordinates, one can easily estimate their modal variances $s t d_{\eta_{j}}^{2}$

$$
s t d_{\eta_{j}}^{2}=\frac{1}{8\left(2 \pi f_{j}\right)^{3} \zeta_{j}} \sum_{i=1}^{N \text { Wind }}\left(\varphi_{R, r_{i}}^{(j)} G_{r_{i} i}\right)^{2} S_{w_{i}}\left(f_{j}\right),
$$

where $f_{j}$ - natural frequency of $j$-th mode shape, $\zeta_{j}-$ dimensionless damping coefficient of $j$-th mode shape, $\varphi_{R, r_{i}}^{(j)}-$ $r_{i}$-th component of $j$-th mode shape, $G_{r_{i} i}$ - load pattern due to $i$-th wind velocity at $r_{i}$-th DOF, $S_{w_{i}}\left(f_{j}\right)$ - value of $i$-th wind fluctuation power spectrum evaluated for $f_{j}$ frequency.

And finally the maximal modal displacement equals

$$
\eta_{j, \max }=\kappa_{j} s t d_{\eta_{j}}
$$

where $\kappa_{j}$ is the peak factor (usually from 3.5 to 5 - after Buchholdt [27]).

Having calculated maximal modal amplitude, maximal physical displacements and stresses can be obtained from Eq. (20) by taking the absolute sum of modal maxima (Petyt [30])

$$
\begin{gathered}
\mathbf{q}_{\max }=\overline{\mathbf{q}}+\sum_{j=1}^{\text {NMode }}\left|\boldsymbol{\varphi}_{R}^{(j)} \eta_{j, \max }\right|, \\
\boldsymbol{\sigma}_{\max }=\overline{\boldsymbol{\sigma}}+\sum_{j=1}^{\text {NMode }}\left|\boldsymbol{\sigma}\left(\boldsymbol{\varphi}_{R}^{(j)}\right) \eta_{j, \max }\right|,
\end{gathered}
$$

where $\overline{\mathbf{q}}$ and $\overline{\boldsymbol{\sigma}}$ stand for mean values of displacements and stresses.

The proposed absolute sum of modal maxima always gives the upper estimation of extreme stresses. This entails that our optimal design, which is sensitive to the assumed constraints, will be on the safe side.

4.3. Continuous minimum weight for the tower. The problem is as follows:

Find fourteen design variables: seven pipe diameters $D_{b}$ and seven their thicknesses $t_{b}$ of structural members, with material density $\rho_{b}$, linked in seven groups, assuring the minimum of the structural volume $V_{c}$ equal to

$$
V_{c}=\sum_{b=1}^{b^{0}} L_{b} A_{b}
$$

Subjected to constraints on:

- Stresses

$$
\left|\sigma_{j, t}\right| \leq \sigma^{0}=215 \mathrm{MPa} \quad j=1,2, \ldots, \text { NElem } .
$$

It is assumed that the largest compressive force $P_{c}$

$$
P_{c} \leq 0.4 P_{E}
$$

- Displacements

$$
\left|q_{\max , i}\right| \leq q_{i}^{0} i=1,2, \ldots, N D O F .
$$

- Maximal and minimal values of pipe diameters and their wall thicknesses

$$
D_{b}^{1} \leq D_{b} \leq D_{b}^{k_{b}^{0}}, \quad t_{b}^{1} \leq t_{b} \leq t_{b}^{k_{b}^{0}}
$$

where $\sigma_{j, t}$ - the largest value of the sum of stresses from bending and tension at the cross section of $j$-th FE, $\sigma^{0}-$ the maximum allowable tension stress, $q_{i}^{0}-$ the maximum allowable displacements. 
The stresses from bending, in members subjected to compressive forces, are multiplied by the amplification factor $\psi=1,6$ arising from constrains (24), (Chen and Atsuta [31]).

The continuous optimization has been performed using MATLAB: Optimization Toolbox and Global Optimization Toolbox. The former contains several types of local minimizers, which allow to solve nonlinear programming problems, but strongly dependent on the initial guess of optimal solution. The latter invokes a selected local minimizer and looks for a global solution using hundreds of different starting points.

Six design variables of the problem have been assigned to cross section parameters of legs and braces for Sec. 1, 2 and 3, respectively. The last variable is related to cross section parameters of all members in Sec. 4 of the tower.

The volume of structural material $V_{c}=0.1803 \mathrm{~m}^{3}$, obtained from continuous optimization is given in Table 1 .

Table 1

Continuous solution

\begin{tabular}{ccccc}
\hline \hline No & $\begin{array}{c}\text { Diameter } \\
{[\mathrm{mm}]}\end{array}$ & $\begin{array}{c}\text { Thickness } \\
{[\mathrm{mm}]}\end{array}$ & $\begin{array}{c}\text { Area } \\
{\left[\mathrm{cm}^{2}\right]}\end{array}$ & $\begin{array}{c}\text { Moment of Interia } \\
{\left[\mathrm{cm}^{4}\right]}\end{array}$ \\
\hline 1 & 48.5 & 2.0 & 2.9217 & 7.9114 \\
\hline 2 & 27.1 & 2.0 & 1.5771 & 1.2499 \\
\hline 3 & 51.9 & 2.1 & 3.2855 & 10.2033 \\
\hline 4 & 46.4 & 2.0 & 2.7897 & 6.8884 \\
\hline 5 & 44.4 & 2.0 & 2.6641 & 6.0000 \\
\hline 6 & 27.9 & 2.0 & 1.6273 & 1.3727 \\
\hline 7 & 41.7 & 2.0 & 2.4944 & 4.9268 \\
\hline \multicolumn{5}{c}{ Total volume $=0.1803 \mathrm{~m}^{3}$} \\
\hline
\end{tabular}

4.4. Discrete minimum weight for the tower. Let us follow now calculations according to the algorithm presented in Sec. 3 .

PART 1: Find values of the discrete cost function.

\section{STEP 1.1}

The continuous optimization presented in the previous Subsec. 4.3 gives $\bar{V}_{c}=0.1803 \mathrm{~m}^{3}$ (Table 1).

\section{STEP 1.2}

The problem with two-branch graph containing all available CSA $A_{b}^{k_{b}}$ and $A_{b}^{k_{b}+1}$, with $I_{b}^{k_{b}}$ and $I_{b}^{k_{b}+1}$, for each of seven linking groups, is constructed (Table 2).

Table 2

Catalogue for graph algorithm

\begin{tabular}{|c|c|c|}
\hline Linking group & $A^{k}[\mathrm{~cm}]$ & $A^{k+1}[\mathrm{~cm}]$ \\
\hline 1 & $2.5384-$ CHS 42.4x2.0 & 3.3238 - CHS 48.3x2.3 \\
\hline 2 & 1.5645 - CHS 26.9x2.0 & 1.7775 - CHS 26.9x2.3 \\
\hline 3 & 3.3238 - CHS $48.3 \times 2.3$ & 4.1362 - CHS 48.3x2.9 \\
\hline 4 & $2.5384-$ CHS $42.4 \times 2.0$ & 3.3238 - CHS 48.3x2.3 \\
\hline 5 & $2.5384-$ CHS $42.4 \times 2.0$ & 3.2509 - CHS 42.4x2.6 \\
\hline 6 & 1.5645 - CHS 26.9x2.0 & 1.9918 - CHS 33.7x2.0 \\
\hline 7 & 1.9918 - CHS 33.7x2.0 & $2.5384-$ CHS 42.4x2.0 \\
\hline
\end{tabular}

STEP 1.3

The closest found, discrete structural volume is $V_{d}=$ $0.1844 \mathrm{~m}^{3}$.
STEP 1.4

The STEP can be omitted, since all possible solutions are found in STEP 1.3.

PART 2. Verifying constraint violation.

\section{STEP 2.1}

The parameter $\mu(1)=\max \left(\left|\sigma_{j, t}\right| \leq \sigma^{0}\right)$ (in this example constraints imposed on displacements are not active) for some of $j^{0}$ structural members are showing that constraints are violated for a value of $V_{d}=0.1844 \mathrm{~m}^{3}$.

\section{STEP 2.2}

We come back to STEP 1.3, looking for the next discrete larger value. After several iteration following value of structural volume fulfilling constrains was found to be: $V_{d}=0.2031 \mathrm{~m}^{3}$.

Comparison of optimal design obtained by full enumeration and present method is presented in Table 3.

Table 3

Comparison of optimal design obtained by full enumeration and present method

\begin{tabular}{|c|c|c|}
\hline Method/Linking group & Full enumaration & Graph algorithm \\
\hline 1 & CHS $48.3 \times 2.3$ & CHS $48.3 \times 2.3$ \\
\hline 2 & CHS 26.9x2 & CHS 26.9x2 \\
\hline 3 & CHS $48.3 \times 2.9$ & CHS $48.3 \times 2.9$ \\
\hline 4 & CHS $48.3 \times 2.3$ & CHS $48.3 \times 2.3$ \\
\hline 5 & CHS $42.4 \times 2.6$ & CHS $42.4 \times 2.6$ \\
\hline 6 & CHS 26.9x2 & CHS $33.7 \times 2$ \\
\hline 7 & CHS $42.4 \times 2$ & CHS $42.4 \times 2$ \\
\hline Total volume $\left[\mathrm{m}^{3}\right]$ & 0.1996 & 0.2031 \\
\hline
\end{tabular}

Since the number of all possible combination for the graph is $2^{7}=128$, it was possible to verify the obtained discrete value applying direct enumeration. Four volumes, fulfilling constraints, are: $0.1996 ; 0.2031 ; 0.2023 ; 0.2058 \mathrm{~m}^{3}$.

It shows that the value obtained by graph algorithm is larger only by less than two per cent of exact value.

\section{Discrete minimum weight design of a floor under synchronized crowd activity}

The second example, showing the applicability of the graph approach to structural optimization is concerning with a floor (Fig. 8) under synchronised crowd activity. The floor is composed of a concrete plate and a system of supporting steel beams.

The example demonstrates the optimization of the floor response for a composite floor in an office environment (Smith et al. [32]). The floor consists of a deep normal weight slab supported by $6.0 \mathrm{~m}$ span secondary beams, which, in turn, are supported by $7.45 \mathrm{~m}$ span castellated primary beams.

The thickness of the plate can be chosen from a catalogue containing five values $h^{p}=[0.10 ; 0.12 ; 0.16 ; 0.18$; $0.20] \mathrm{m}$. On the other hand, there are four design variables for beams. All of them are selected from a catalogue (British Universal Columns and Beams), containing 55 different sets of cross section areas and related to them moments of inertia and sectional moduli with respect to two axes, starting from $A^{1}=16.5 \mathrm{~cm}^{2}$ and ending at $A^{55}=228.1 \mathrm{~cm}^{2}$. 


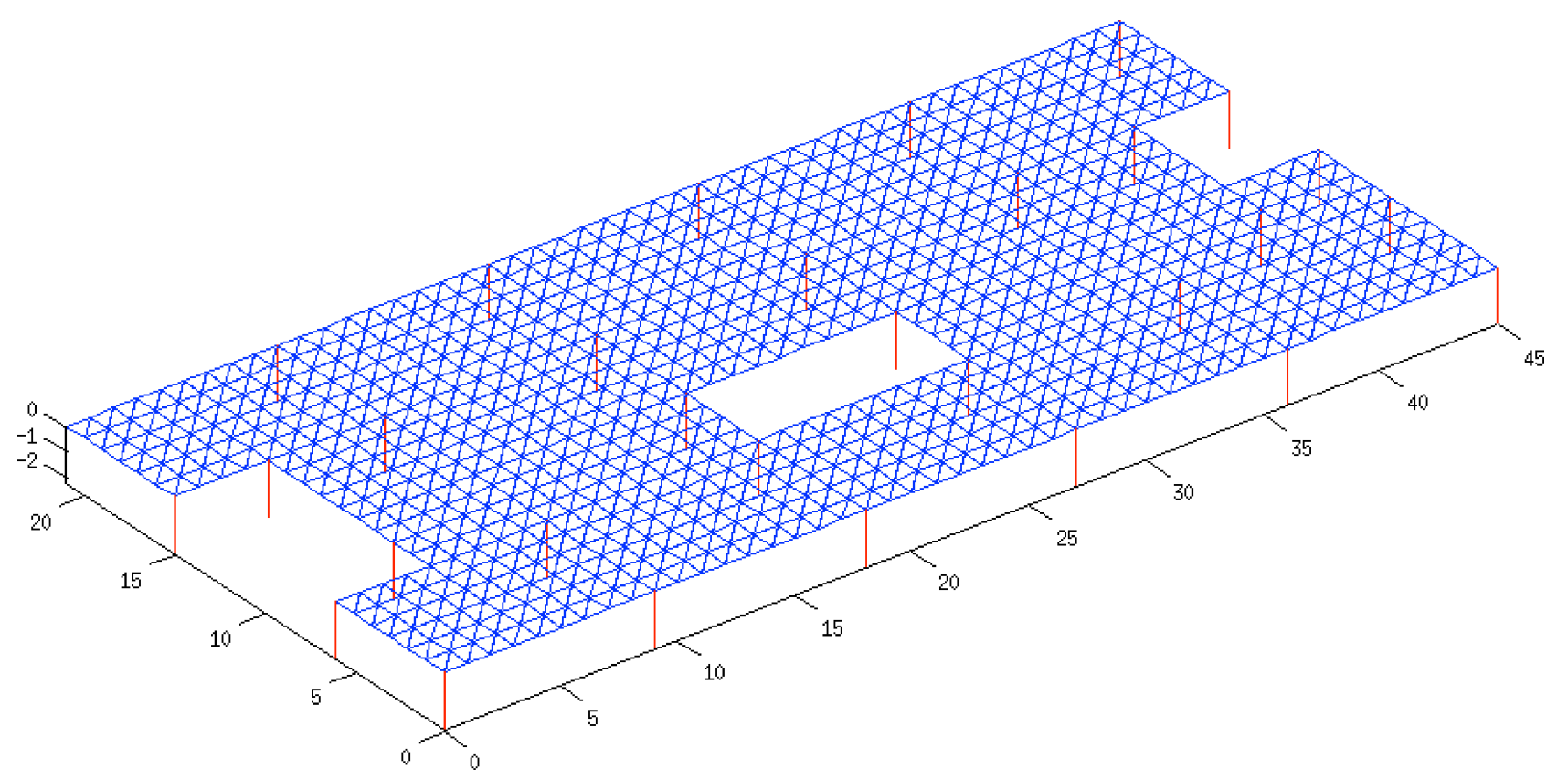

Fig. 8. Composite floor supported by steel beams

5.1. Deterministic load from synchronised crowd activity and structural response. In the case of a floor under synchronized crowd activity, the load-time function can be expressed by a sequence of semi-sinusoidal pulses; this aligns well with measurements for individuals (Smith et al. [32])

$$
p_{\text {jump }}(t)=Q\left\{1.0+\sum_{h=1}^{H} \alpha_{h} D_{\delta, h}^{(1)} \sin \left(2 h \pi f_{p} t+\theta_{h}+\theta_{h}^{(1)}\right)\right\} \text {, }
$$

where $Q$ is the weight of the jumpers (usually taken as $746 \mathrm{~N}$ per individual), $H$ is the number of Fourier terms, $\alpha_{h}$ is the $h$-th Fourier coefficient (or dynamic load factor), $f_{p}$ is the frequency of the jumping load $(\mathrm{Hz}), \theta_{h}$ is the phase lag of the $h$-th term, $\theta_{h}^{(1)}$ is the phase of the response of the $1^{\text {st }}$ mode relative to the $h$-th harmonics.

The right side of Eq. (7), for the case of the floor, is taking the following form

$$
\begin{gathered}
\boldsymbol{\Phi}_{R}^{T} \mathbf{p}(t)=\boldsymbol{\Phi}_{R}^{T} \mathbf{B} p_{\text {jump }}(t) \\
=\boldsymbol{\Phi}_{R}^{T} \mathbf{B} Q\left\{1.0+\sum_{h=1}^{H} \alpha_{h} D_{\delta, h}^{(1)} \sin \left(2 h \pi f_{p} t+\theta_{h}+\theta_{h}^{(1)}\right)\right\}
\end{gathered}
$$

where $\mathbf{B}$ is Boolean matrix which nonzero elements correspond to jumpers location.

The $j$-th component of (9) can be presented as

$$
\begin{gathered}
\ddot{\eta}_{j}(t)+2 \zeta_{j} \omega_{j} \dot{\eta}_{j}(t)+\omega_{j}^{2} \eta_{j}(t) \\
=\boldsymbol{\phi}_{R}^{(j)^{T}} \mathbf{B} Q\left\{1.0+\sum_{h=1}^{H} \alpha_{h} D_{\delta, h}^{(1)} \sin \left(2 h \pi f_{p} t+\theta_{h}+\theta_{h}^{(1)}\right)\right\} .
\end{gathered}
$$

Applying known solutions for linear vibration problems, we get the acceleration at the point of maximal modal deformation, for the first mode (Fig. 9) and $h$ harmonic

$$
\ddot{q}_{j, h}^{R M S}=\varphi_{\max , 1}^{(j)} \varphi_{\max , 1}^{(j)} \frac{\ddot{q}_{h} Q}{\sqrt{2}} D_{a, h}^{(j)},
$$

where

$$
D_{a, h}^{(j)}=\frac{\left(h \beta_{j}\right)^{2}}{\sqrt{\left(1-h \beta_{j}\right)^{2}+\left(2 \zeta_{j} h \beta_{j}\right)^{2}}}
$$

is the dynamic magnification factor for acceleration. The maximum acceleration is found from summation

$$
\ddot{q}^{R M S}=\sum_{j=1}^{\text {NMode }} \sum_{h=1}^{H} \ddot{q}_{j, h}^{R M S}
$$

Similarly, displacement at the point of maximal modal deformation, for the first mode and $h$-th harmonic

$$
\begin{gathered}
q_{j, h}^{R M S}=\varphi_{\max , 1}^{(j)} \varphi_{\max , 1}^{(j)} \frac{\alpha_{h} Q}{\sqrt{2}} D_{\delta, h}^{(j)}, \\
D_{\delta, h}^{(j)}=\frac{1}{\sqrt{\left(1-h \beta_{j}\right)^{2}+\left(2 \zeta_{j} h \beta_{j}\right)^{2}}}
\end{gathered}
$$

and the maximum displacement

$$
q^{R M S}=\sum_{j=1}^{\text {NMode }} \sum_{h=1}^{H} q_{j, h}^{R M S}
$$




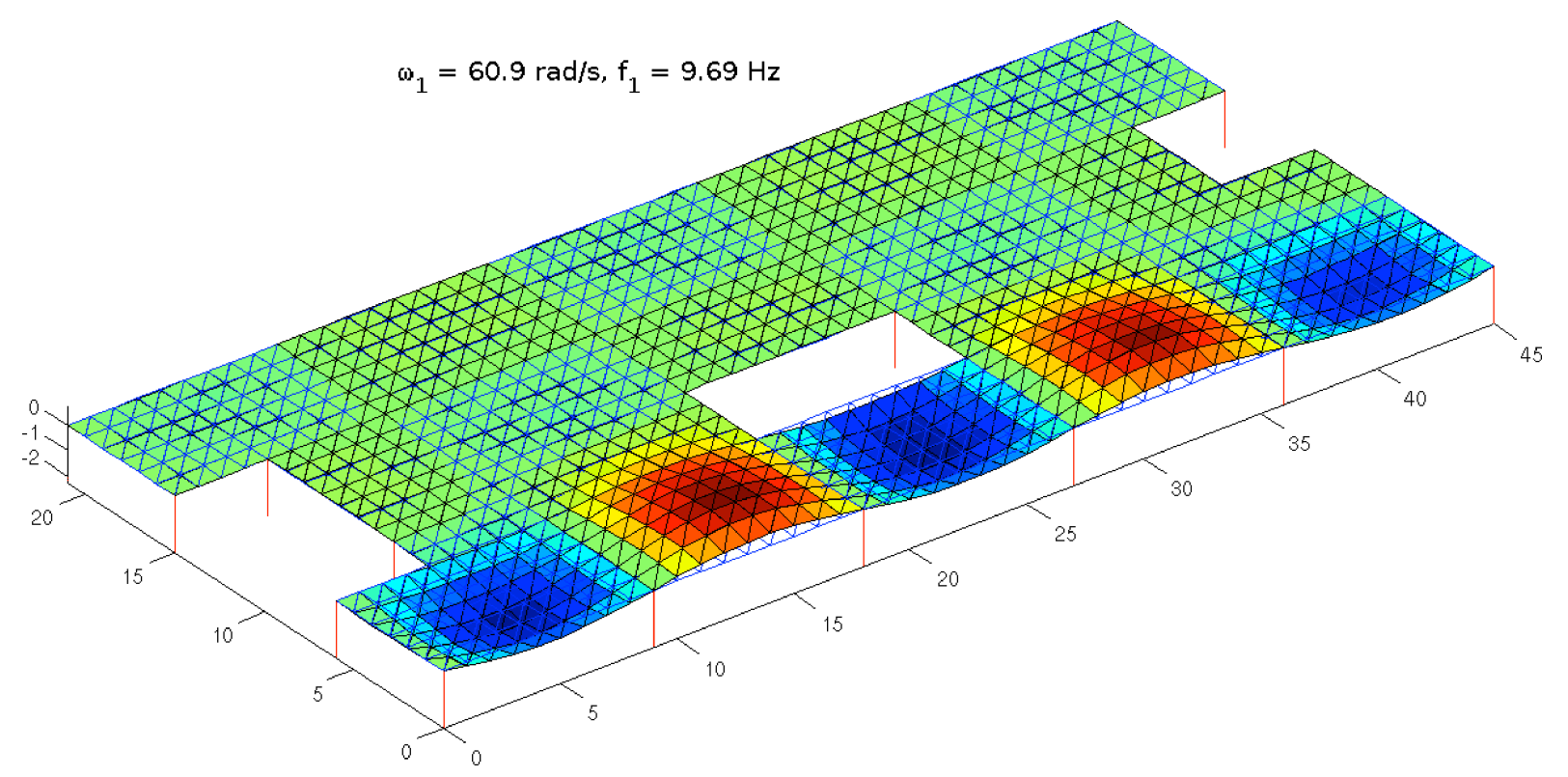

Fig. 9. First mode shape of composite floor

5.2. Continuous minimum weight for the floor. The problem consists in finding six design variables, assuring the minimum of the structural weight $W_{c}$ equal to (notations in Sec. 3)

$$
W_{c}=\rho_{b} \sum_{b=1}^{b^{0}} L_{b} A_{b}+\rho_{p} h_{1} S_{1},
$$

where $\rho_{b}$ is the mass density of the beam material, $\rho_{p}$ is the mass density of the floor composite.

Subjected to constraints on:

- Displacements

$$
q^{R M S} \leq q_{\text {allowable }}
$$

- Accelerations

$$
\ddot{q}^{R M S} \leq \ddot{q}_{\text {allowable }}
$$

- Maximal and minimal values of floor composite thickness

$$
h_{p, \min } \leq h_{p} \leq h_{p, \max }
$$

- Maximal and minimal values of the cross section area of beams

$$
A_{b}^{\min } \leq A_{b} \leq A_{b}^{\max }, \quad b=1,2,3, \ldots, b^{0}
$$

and related to CSA height $H_{b}$ of I beam and its web $h_{b}$.
Similarly to the tower problem, continuous optimization has been performed using MATLAB: Optimization Toolbox and Global Optimization Toolbox.

Four design variables of the problem are assigned to cross section areas, and related to them moments of inertia, of supporting beams and one to the plate thickness.

The distance between the plate middle surface and centroid of the beam cross section (eccentricity), for the largest profiles is assumed to be

$$
e=\frac{h_{b}}{2}+\frac{h_{p}}{2}
$$

State constrains are imposed on acceleration $\ddot{q}^{R M S} \leq 0.6 \mathrm{~m} / \mathrm{s}^{2}$ (Smith at al. [32]) and equivalent static stresses, which are calculated for the following forces

$$
p_{\text {jump, equiv }}=Q\left(1.0+\sum_{h=1}^{H} \alpha_{h} D_{\delta, h}\right) .
$$

For beams allowable stress equals to $\sigma_{b}^{0}=275 \mathrm{MPa}$ and

\begin{tabular}{|c|c|c|c|}
\hline Linking group & Continuous Solution & Discrete two-branch graph for beams and plate & Discrete solution $D_{b}$ \\
\hline 1 plate & 250.00 & $\{250.00,300.00\}$ & $\begin{array}{c}250.00 \\
(\text { Slab thickness }=\mathbf{1 0} \mathbf{~ c m})\end{array}$ \\
\hline 2 beam & 58.88 & $\{56.99,59.82\}$ & $\begin{array}{c}59.82 \\
\text { (UB 457x152x60) }\end{array}$ \\
\hline 3 beam & 164.07 & $\{149.15,179.06\}$ & $\begin{array}{c}179.06 \\
\text { (UB } 610 \times 305 \times 179)\end{array}$ \\
\hline 4 beam & 108.33 & $\{101.19,109.04\}$ & $\begin{array}{c}109.04 \\
\text { (UB 533x210x109) }\end{array}$ \\
\hline 5 beam & 65.94 & $\{60.05,67.12\}$ & $\begin{array}{c}60.05 \\
\text { (UB 533x210x60) } \\
\end{array}$ \\
\hline Structural weight $(\mathrm{kg})$ & 251780 & $\{249330,295570\}$ & 252282 \\
\hline
\end{tabular}
for concrete of the plate $\sigma_{p}^{0}=35 \mathrm{MPa}$.

The weights $\rho_{b} v_{b}$ and $\rho_{p} v_{p}$ of structural members linked in the five groups, obtained from continuous optimization are given in Table 4.

Table 4

Continuous, optimal CSA and plate thickness: available parameters for two-branch graph and discrete optimal solution 
5.3. Discrete minimum weight for the floor. PART 1: Find values of the discrete cost function.

\section{STEP 1.1}

The weights $\rho_{b} v_{b}$ and $\rho_{p} v_{p}$ of structural members linked in the five groups, obtained from continuous optimization in section 5.2, are given in Table 4.

\section{STEP 1.2}

The lists of two available beams $\rho_{b} A_{b}^{k_{b}}$ and $\rho_{b} A_{b}^{k_{b}+1}$ for each linking group and two available thicknesses of the plate $\rho_{p} h_{p}^{k_{p}}$ and $\rho_{p} h_{p}^{k_{p}+1}$, are constructed. They are listed in Table 4 .

\section{STEP 1.3}

For assumed $m=3$, permutations the listed weights are randomly selected.

\section{STEP 1.4}

Performing calculation (i)-(v), a set of $m=3$ discrete values of the cost function are found. They are: $W_{d}=$ $\{252178 ; 252282 ; 291980\} \mathrm{kg}$.

PART 2.: Verifying constraint violation.

Verification of all constrains shows that for the smallest weight, they are violated. In the case of the second weight $W_{d}=252282 \mathrm{~kg}$, all constrains are satisfied. It is then assumed that this is the discrete minimum weight solution. Values of optimum parameters for each structural member are listed in Table 4.

\section{Conclusions}

A DSO algorithm for structures composed of prefabricated elements, subjected to stochastic and deterministic loads, is proposed. The algorithm can be applied to problems with very large numbers of combinations, arising from numbers of structural elements and available parameters.

The algorithm is based on a heuristic assumption, that the structural weight obtained from continuous solution, constitutes a lower bound for a discrete minimum weight. This assumption, together with the graph representation of the structural volume, permits to reject from considerations, very large numbers of structures with volumes smaller, than the volume obtained from a continuous solution. The algorithm is relatively easy to apply. The only knowledge needed is the FEM and graph representations. In the evolutionary type of algorithms, the designer, has to decide about: number of chromosomes, number of generations, which of six known methods of the selection of chromosomes for a crossover should be taken, which of six or even more mutation types should be applied, the penalty coefficient. There are no general rules of applying, this or other set of the mentioned algorithm components. The decision has to be made through many tedious numerical experiments. Moreover, evolutionary algorithms need tens of thousands, or even more, of analysis. The numbers come from products of numbers of chromosomes multiplied by numbers of generations.
The proposed algorithm is numerically very efficient. It requires relatively small numbers of dynamic analyses, comparing with Evolutionary Optimization type algorithms [33].

The presented examples are showing advantages of the proposed algorithm. The number of dynamic analyses, needed to verify, whether obtained from the graph solutions are not violating constraints, is small. In the first example, with the transmission tower, this number is 9 . In the second one, dealing with the composite floor, supported with steel beams, this number is 3 . The number of analysis in the continuous optimisation depends upon the assumed number of starting points, however usually it does not exceed hundreds.

Acknowledgements. The support of the Polish National Science Centre, Grant No 0494/B/T02/2011/40 is much appreciated.

\section{REFERENCES}

[1] M.J. Turner, "Design of minimum-mass structures with specified natural frequencies", AIAA J. 5 (3), 406-412 (1967).

[2] K.C. Tang, M.Q. Brewster, E.J. Haug, B.R. McCart, and T.D. Streeter, "Optimal design of structures with constraints on natural frequency", AIAA J. 8 (6), 1012-1019 (1970).

[3] Z. Mroz, "Optimum design of elastic structures subjected to dynamic, harmonically-varying loads", Ang. Math. Mech. 50, 303-309 (1970).

[4] B.L. Pierson, "A survey of optimal structural design under dynamic constraints", Int. J. Numerical Methods in Engineering 4 (4), 491-499 (1972).

[5] O.G. McGee and K.F. Phan, "On the convergence quality of minimum-weight design of large space frames under multiple dynamic constraints", Structural Optimization 4, 156-164 (1992).

[6] R. Grandhi, "Structural optimization with frequency constraints - a review", AIAA J. 31 (1870), 2296-2303 (1993).

[7] J.S. Arora, "Methods for discrete variable structural optimization", in Recent Advances in Optimal Structural Design, ed. S.A Burns, pp. 1-40, ASCE Publication, Reston, 2002.

[8] L.C. Lee, B. Castro, and P.W. Partridge, "Minimum weight design of framed structures using a genetic algorithm considering dynamic analysis", Latin American J. Solids and Structures 3, 107-123 (2006).

[9] W. Gutkowski, "Structural optimization with discrete design variables", Eur. J. Mech., Solids A 16, 107-126 (1997).

[10] B. Blachowski and W. Gutkowski, "Discrete structural optimization by removing redundant material", Engineering Optimization 40 (7), 685-694 (2008).

[11] B.S. Kang, W.S. Choi, and G.J. Park, "Structural optimization under equivalent static loads transformed from dynamic loads based on displacement", Computers and Structures 79, 145-154 (2001).

[12] W.S. Choi, K.B. Park, and G.J. Park, "Calculation of equivalent static load and its application", Trans. SMIRT Washington DC 16, paper \#1111 (2001).

[13] M. Papadrakakis, N.D. Lagaros, and V. Plevris, "Multiobjective optimization of skeletal structures under static and seismic loading conditions", Engineering Optimization 34 (6), 645-669 (2002). 
[14] A. Norkus and R. Karkauskas, "Truss optimization under complex constraints and random loading", J. Civil Engineering and Management 10 (3), 217-226 (2004).

[15] H.A. Jensen and M. Beer, "Discrete-continuous variable structural optimization of systems under stochastic loading", Structural Safety 32 (5), 293-304 (2010).

[16] H.Y. Guo and Z.L. Li, "Structural topology optimization of high-voltage transmission, tower with discrete variables", Structural and Multidisciplinary Optimization 43 (6), 851-861 (2011).

[17] W. Gutkowski, J. Bauer, and Z. Iwanow, "Support number and allocation for optimum structure", Discrete Structural Optimization, Proc. IUTAM Symp. 1, 168-177 (1993).

[18] Z. Iwanow, "An algorithm for finding an ordered sequence of values of a discrete linear function", Control and Cybernetics 6, 238-249 (1990).

[19] B. Blachowski and W. Gutkowski, "A hybrid continuousdiscrete approach to large discrete structural optimization problems", Structural and Multidisciplinary Optimization 41 (6), 965-977 (2010).

[20] B. Blachowski and W. Gutkowski, "Revised assumptions for monitoring and control of 3D lattice structures", $11^{\text {th }}$ PanAmerican Congress Applied Mechanics PACAM XI 1, CDROM (2010).

[21] J. Gu, Z.D. Ma, and G.M. Hulbert, "A new load-dependent Ritz vector method for structural dynamics analyses: quasistatic Ritz vectors", Finite Elements in Analysis and Design 36 (3), 261-278 (2000).

[22] B. Blachowski, "Model based predictive control of guyed mast vibration", J. Theoretical and Applied Mechanics 45 (2), 405423 (2007).
[23] W. Borutzky, Bond Graph Methodology: Development and Analysis of Multidisciplinary Dynamic System Models, SCS Publishing House, Erlangen, 2004.

[24] A. Buchacz, "Modifications of cascade structures in computer aided design of mechanical continuous vibration bar systems represented by graphs and structural numbers", J. Materials Processing Technology 157-158, 45-54 (2004).

[25] J.A. Bondy and U.S.R. Murty, Graph Theory, Springer, Berlin, 2008.

[26] E. Wilson, Three-Dimensional Static and Dynamic Analysis of Structures, $3^{\text {rd }}$ edition, Computers and Structures Inc., Berkeley, 2002.

[27] H.A. Buchholdt, Structural Dynamics for Engineers, Thomas Telford, London, 1997.

[28] L. Carassale and G. Piccardo, "Double modal transformation and wind engineering applications", J. Engineering Mechanics 127 (5), 432-439 (2001).

[29] E.N. Strommen, Theory of Bridge Aerodynamics, Springer, Berlin, 2006.

[30] M. Petyt, Introduction to Finite Vibration Analysis, $2^{\text {nd }}$ edition, Cambridge University Press, Cambridge, 2010.

[31] W. Chen and T. Atsuta, Theory of Beam-columns, Space Behavior and Design, vol. 2, J. Ross Publishing, London, 2008.

[32] A.L. Smith, S.J. Hicks, and P.J. Devine, "Design of floors for vibration: a new approach", The Steel Construction Institute, New York, 2009.

[33] M. Szczepaniak and T. Burczynski, "Swarm optimization of stiffeners locations in 2D structures", Bull. Pol. Ac.: Tech. 60 (2), 241-246 (2012) 